\title{
Neue Versionen der Riote du Monde.
}

I.

Das Stück der Hss. von Bern und Epinal.

Es steht a) in der Hs. 205 der Berner Stadtbibliothek auf fol. I $17^{\mathrm{r}}-118^{\mathrm{v}}$, trägt den Titel: S'ensuyt la nature de l'ome et ses bonnes condicions und zählt I 33 Verse.

b) in der Hs. 189 von Epinal auf fol. $4 \mathrm{I}-43$ (cf. die Beschreibung dieser Hs. durch F. Bonnardot in: Bulletin de la Société des anciens textes français 1876, S. 84), trägt den Titel: Item, contre pluseurs gens, zählt i I Verse mehr als in Hs. B, während ihm anderseits einige Verse fehlen, die in B stehen.

Ich gebe den Text nach $B$, füge die Verse von $E$ in Klammern bei und notiere Abweichungen von $\mathrm{E}$ wie Besserungsvorschläge unten. Rein orthographische Varianten sind spärlich mitgeteilt. Ich mache darauf aufmerksam, dafs meine Lesarten von $\mathrm{E}$ nicht immer mit denen Bonnardot's stimmen. V. I 8 liest B. hutez für hurez, was schon Godefroy unter huré gebessert hat.

Ia.

On m'a maintefoyz demandé

Que c'est [ne] qui m'a destourné

A maintenir si com je sueil;

Ne scevent pas dont je me dueil.

5 Mais je croy bien, s'ilz le savoient,

Que pour excusé me tendroient.

Nul ne scet faire contenance

Qui a chascun viengne a plaisance.

Je voy que, s'uns homs est preudons,

Io On dit que c'est ung droit moutons.

Et s'il est bons simples tenuz,

On dit que c'est ung droit Jesus.

Et s'il est sages et soubtilz:

Haro! qu'il a de mal apris!

I5 Et s'il est preudons et viseux,

On dit qu'il est malicieux.

I on m'a $B$ en ant (ant durchgestrichen) $E \quad 2$ ne $E$ destournez $E$ 3 si com je sueil] ce que je seul $E$, deul $E$ c'il $E$ excusez $E$ I1. 12 fehlen $E$ I4. I5 umgestellt $E$ 
S'il est chauve, c'est ung pelé;

S'il a grant col, c'est ung huré.

S'il a lonc nez, c'est ung becus;

20 S'il a court, c'ess ung camus.

S'il est espes, ung champion;

S'il est tendres, ung vession.

S'il est pensuz, c'est ung trippier;

S'il est maigres, c'est ung levrier.

25 S'il est riches, c'est ung merchant;

S'il est povres, c'est ung truant.

S'il est rez a rez chascun an:

Noant plus n'a il ouan (plus) qu'antan.

S'il gaste le sien ou despent,

30 On dit qu'il ne tient bram ne vent.

[S'il est large: c'est tout gastez;

S'il est eschars: c'est bien merdez.]

S'il boit voulentiers ou il feste,

On dit qu'il vit comme une beste.

35 Et s'il est larges viandier:

C'est son dieu que boire et mengier.

Et s'il veut vivre par raison:

On meurt de fain en sa maison.

Et s'il va souvent oür messe,

40 On dit: par ypocrissie esce;

Qu'il fait le beguin papelart.

Et s'il i va ou tempre ou tart,

On dit qu'il vient de mauvais lieu;

C'est ung bougre, il ne croit dieu.

45 Et s'il ayme les compaignons

Ou va souvent en lour maison[s]

Et du sien paie et riens dou leur,

On dit qu'il ressemble au jougleur,

Qui n'a pis fors qu'en son hostel.

50 Et s'il n'y va, on redit el:

[On dit que c'est ung droit couvaux,

Que couve pour cez yeulfz tenir chaux.]

S'i! va droit, on dit qu'il s'estant;

S'il va courbe: il va contre vent.

55 S'il marche tost: com il s'affiche!

Dieu! quil fait le gros et le riche!

S'il entre coyement en l'uis:

17 chauve $E$ chanuz $B \quad$ I 8 col $B$ toupe $E \quad 22$ tendres $B$ tenves $E$ vession $B$ vetion $E$ corr. vre (= vrai) scion? 27 s'il est rez a rez $B$ sil a rest a rest corr. aus etwas anderem 28 noant $B$ nen $E$ plus fehlt $E$ 29 ou $B$ et $E \quad 30$ bram $B$ bren $E \quad 31$ boit $B$ boy $E \quad 37$ fehlt $E$ 40 par ypocrissie esce (= est ce) $B$ que c'est ypocrisesse $E 42$ i $B$ ny $E$ 44 croit $B$ croit en $E$, vielleicht vorsusiehen 46 ou $B$ et $E$ 49 qui $B$ que $E \quad 53$ fehlt $E \quad 55$ s'il marche tost $B$ s'il passe fort $E$

Zeitschr. f. rom. Phil. XXIV. 
Il semble qu'il ait les piez cuiz.

S'il parle attrait: c'est ung liegaux;

60 S'il se taist: c'est ung droit uniaux.

S'il parle hault a tost parler:

In ne fera que plaidoier.

S'il chante bien: c'est ung jougleur;

S'il dit beaux moz: c'est ung trouveur.

65 S'il ne chante ne esbanie:

Il ne vault riens en compaignie.

S'il est honteux et peu parliers:

Il n'est ne Hue ne Gaultiers.

S'il se vest bien et cointement:

70 Il est si cointes qu'il se pent.

S'il ne se vest bien et souvent:

Il se maintient trop nicement.

S'il se chausse estroit par raison:

Il mect ses deux piez en prison.

75 S'il a grans soulers, on le moque:

C'est ung vilain, ung choqueroque.

S'uns homs mangue bien o boit:

Celluy la roffle quanqu'il voit.

S'au boire n'au mangier n'est preux,

80 On dit qu'il est trop dongereux.

S'il est souvent tempre couchant,

On dit que c'est ung dieu dormant.

Et se le dormir ne ly haite:

Il ne dort point, c'est une gaite.

85 S'il gist en son lit estanduz,

On dit qu'il ressemble ung panduz.

[S'il rit souvent: c'est ung droit sot;

C'est ung mellin, c'il ne rit tost.

S'il (n')a mie: il enraige tout vif!

90 Et c'il n'a mie: c'est ung chetif.

Se ungs hons se met en mariage,

On dit qu'il ait ou corps la rage.]

S'il a bonne: c'est mescheance;

S'il a belle: il [est] en doubtance.

95 Et s'il a mauvaise assenee:

Il a biens le deable espousee.

S'il ne se veult marier point:

Il n'en puet trouver nulle a point.

59 liegaux $B$ nigaux $E \quad 60$ uniaux $E$ ajuyaux $B \quad$ 6I nach $B$ S'il parle tos[t] e[t] qu'es[t] grant parlier $E$ 62 nach $B$ Ja ne finera de pladier $E 63$ jougleur $E$ jougloux $B \quad 65$ peu $B$ poc $E$ il n'est $B$ ce n'est $E \quad 71$ bien $B$ bel $E \quad 76$ choqueroque $E$ chocreroque (P) $B \quad 77$ mangue $B$ manjut $E \quad 78$ roffle $B$ rifle $E$ 80 dongereux $B$ dangereux $E$ 83 ly $B$ luy $E \quad 84 B$ dor $E \quad 86$ panduz $B$ pendut $E$ 93. 94 a $B$ $l^{\prime 2} E 96$ bien fehlt $E 98$ il n'en $B$ ne ne $E$ 
Ib.

Mais encores me fait il pis,

100 Car on parle sur les païs.

Je voys a dextre et a senestre,

Je ne sçay de quel païs estre.

S'il est François, malicieux;

S'il est Picart, trop enuyeux;

I05 S'il est ort, c'est ung Alement,

Et grant buveur, s'il est Norment,

Et jureur, s'il est Bourguignon,

Et trop testu, s'il est Breton.

Fort a cognoistre: c'est Anglois;

I 10 S'il est Escot, trop felonnois.

S'il est Provenc(i)al, enquereur:

S'il est Lombart, il joue aux dez.

S'il est Romain, trop couvoiteux;

C'est Espaignot, luxurieux.

115 Et pour ce je me passe a tant,

Que je [ne] vous voise ennuy(s)ant.

II.

Mais j'ose dire en verité

Que, quant on a par tout esté

Et essayé le grant mesaise,

I 20 N'est il vie que d'estre aise

$\mathrm{Ne}$ maladie que de corps

Ne si grant destresse que mors

$\mathrm{Ne}$ bonté qui vaille de l'ame

[Ne beauté que de belle dame]

I25 Ne sens qui vaille cellui d'omme

$\mathrm{Ne}$ dormir fors quant on a somme

Ne meschief fors que d'avoir fain

$\mathrm{Ne}$ dangier fors que de villain

$\mathrm{Ne}$ noblesse que de donner

$130 \mathrm{Ne}$ merderie que d'aver

$\mathrm{Ne}$ don qui vaille de courtois

$\mathrm{Ne}$ chanter que de bonne voix

$\mathrm{Ne}$ deduit qui vaille d'amant

$\mathrm{Ne}$ d'errer fors que par beau temps

99 encores $B$ encor $E \quad 100$ car $B$ con $E$ wohl $z u$ ändern in quant ror voys $E$ vif $B \quad 104$ enuyeux $E$ enuieux oder envieux $B$ 106 s'il $B$ il $E$ 107. 108 s'il $B$ cil $E$ 109 c'est $B$ c'est ung $E$ I I I Provencial $B$ Prouvencel $E$ III. II2 $B$ hat enquereur : joue aux dez $E$ hat enquerrier : pere au deniere enquerrier ist eine unmögliche Form. Sind Nominative herzustellen: enquerere : au dé jouere? ${ }^{1} 4$ Espaignot $B$ Espaignoil oder Espaignois $E$ I15 me $B$ m'en $E$ I16 ne fehlt $B$ ennuysant $B$ ennuiuant $E$ II8 que quant] car quant $B$ que que $E$ irg le $B$ la $E$ 120 n'est $B$ se n'est $E$ 122 mors $B$ de mors $E$ 129. 130 umgestellt $E$ 133 amant $B$ amanz $E \quad 134$ errer] desirer $B$ d'aller corr. aus d'errer $E$ 
I35 Ne joieux fors que d'estre lié

$\mathrm{Ne}$ travail que d'aler a pié.

Ne rien qui vaille bonne fin

Et dieu amer de bon cuer fin,

Autrui amer an charité

I40 Autant com soy en verité.

Qui ce fera parfeitement,

Se l'escripture ne nous ment,

En la fin aura paradis;

La nous maint le doulz Jesucrist.

Amen.

Explicit pulcra et gratiosa dicta.

Scriptor qui scripsit cum Cristo vivere possit.

II.

Das Stück von Metz 855 fol. $8^{\text {b. }}$.

Ung doctour cedenucusse. (rot)

[8b] Qui est celui qui ne doie troubler, estre iriez et courouciez, I. quant il voit lez vertus ordoiez per crimenacion et per murmure?

Se ung homme est humble, il est appelleit de pluseurs vil et homme 2 . de nyant. orguilleux.

Se ung homme s'eslieve per raison et per justice, on dit qu'il est 3 .

Se ung homme est moings saige per sa nonsaichance, on cuide 4 . c'on se doit de lui moqueir et rire.

Se ung homme est rigoreux et penissant (sic) les malvais, il est 5 . tenus pour crueil.

Se ung homme perdonne per pitié les malz et les pechiez a ceulx 6 . qui se repentent, il est blasmei et reprin[s] de faulsetei, et dit on qu'il [done] occoison de faire plus de malz, de pechiez et de legiereteis. honorei.

Se ung homme est simple, on le despite comme bestial et a des- 7 .

Se ung homme est aigre et appert en son droit soustenir, on le fait 8 . comme homme bareteur et plain de malvistié.

Se ung homme est diligent, on dit qu'il est plain de crueuseteis. 9.

Se ung homme laist son droit a soustenir, on dit qu'il est negli. Io. gent et nice.

Se ung homme est saige en faire sez besoingnes et cleirvoiant, on Ir. dit qu'il est covoiteux et d'acquerir ardant.

[8c] Se ung homme est repons, on dit qu'il est laisse et pereseux. 12.

Se ung homme est soubre et abstinent, on dit qu'il est eschars et 13 . avaricieux.

Se ung homme vit honestement et largement, on dit qu'il est glouton I4. et diffameis.

I35 joieux $B$ desir $E 136$ d'aller $B$ deller $E$ corrigiert aus derrer 135. 136 umgestellt $E \quad 140 \mathrm{com} B$ comme $E$ verite $B$ amytez $E$ 144 maint $B$ doint $E$. 
Je ne cuide mie qu'il ait personne a monde vivant que plaice a tous; et dit: Fais bien tant comme tu vis et ne fais force dez parolles des malvais.

Ce sont les auctoritez de S'. et de grant maistre. (rot)

Il nait (sic) dessoubz le ciel si belle ne si bonne compaignie come 16. celle de paradis.

Il n'est dessoubz le ciel si bonne clergie comme de apprendre a 17. morir et savoir vivre.

Il n'est dessoubz le ciel si bonne chevalerie come d'acquerir vertus 18 . et vaincre lez vices.

Il n'est dessoubz le ciel si grande courtoisie come de son cuer bien 19. gardeir et affaitier.

Il n'est dessoubz le ciel si beaul royaulme come estre roi et sire 20 . de soi.

Il n'est dessoubz le ciel si beaul mestier comme de dieu servir, 21 . loer et merciier.

Il n'est dessoubz le ciel ( $m s$. dieu) si grande franchise comme d'estre 22 . dessoubz dieu tant soulement.

$I 1$ n'est dessoubz le ciel ( $m s$. dieu) si grande phi[8 d $]$ losophie comme 23. de soi congnoistre vraiement.

Il n'est dessoubz le ciel si grande seignorie ne joie ne soulas ne 24 . doulceur ne bonne vie comme de dieu ameir perfaitement.

C'est la fin de nostre pelerinaige. Pour ce est homme fait et a ce 25 . doit il entendre. Faus est le pelerins qui de la fin de sa voie ne sceit nyant ne nyant rien enquiert.

\section{III.}

\section{Das Fragment von Paris.}

Dasselbe steht auf dem Einband der Hs. 7609 fonds français der Nationalbibliothek und ist von Fr. Michel in seiner Riote du Monde (Paris 1 834) pp. 44-45 abgedruckt worden. Bei der Seltenheit dieses Opusculums wird es am Platze sein, die 35 oder 37 Verse hier abdrucken zu lassen.

[Car quant on a partout esté]

Et esprovir biens et mesaise,

N'est il un fors que d'estre aise.

Bonté ne de a ries tant k'a l'ame

5 N'est biautés fors de bele dame

N'est sens qui vaille celui d'oume.

N'est dormirs fors quant on a somme.

N'est maladie fors de cors.

N'est si grant destrece que mors.

Io N'es[t] mengier fors quant on a fain.

N'est dangiers for $[\mathrm{s}] \mathrm{ke}(\mathrm{s})$ de vilain.

2 corr. Et esprové 3 un] corr. vie 4 ne de a ries] corr. n'ede a riens 


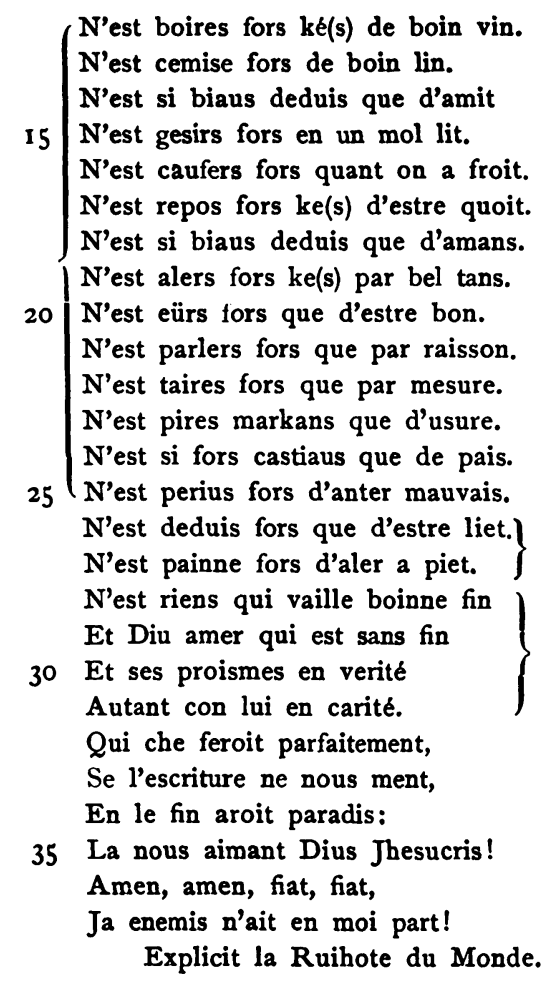

IV.

Die Hs. 189 von Epinal, von der ich unter I. redete, enthält auf fol. $70^{\circ}$ folgende vier Verse nach allerlei Sprüchwörtern:

Nulle riocte n'entreprendes, je te prie,

A ton pareil(le), car chose est perilleuse.

A plus grant de toy c'est oultrage et folie;

A mendre et a foble est chose honteuse.

V.

Wenn ich die Ztschr. VIII 275-289 und im Vorangehenden veröffentlichten Texte übersehe, ergeben sich folgende Teile dieses Spielmannsgedichts: 1

35 aimant] corr. maint

1 Die Sigel bedeuten: $B=$ Bern 113 .

$B_{1}=$ Bern 205.

$\mathrm{C}=$ Cambridger Trinity College O. 2. 45 .

$\mathrm{E}=$ Epinal 189 .

$\mathbf{H}=$ Harleian 2253.

$\mathbf{M}=$ Metz 855 .

$\mathrm{P}=$ Paris Nationalbibliothek 1553 fonds fr.

$\pi=$ Paris Nationalb. 7609 (Einband). 
A. I. Le jongleur et le roi:

II. La vie du jongleur:

III. La riote du monde:

IV. Ernsthafte Lehre des Jongleurs an den König:

V. Burleske Unterhaltung des Jongleurs mit dem König; des erstern Sprüche als Abweiser

B. I. La riote du monde

II. Les proverbes ethniques III. Les verités $\alpha$ ) la ballade:
Reimversion vv. $\mathrm{I}-142$ (H),

Prosaversion $1-36$ (PC).

Reimversion vv. 143-I 88 (H),

Prosaversion 36-48 (PCB).

Reimversion vv. 189-379 (H),

Prosaversion 49-7 I (PCB).

Reimversion vv. 380-405 (H).

Prosaversion 72-IOI (PCB),

Fragment von Epinal =

Prosaversion $n^{0} 4 \mathrm{I}$ (E).

Reimversion vv. $I-98\left(B_{1} E\right)$,

Prosaversion I-I6 (M).

Reimversion vv. 99-II6 (B $\left.B_{1} E\right)$.

I) Reimversion vv. II 7-I $44\left(B_{1} E\right)$,

2) Fragment von Paris $(\pi)$.

$\beta)$ Texte religieux: Prosaversion 17-26 (M).

A I bildete zunächst ein Stück für sich. Die Sammlung von Wortspielen findet sich häufig, so in dem Mystère: Saint Cristophe, von Chevalet, in dem Pédant joué von Cyrano de Bergerac, im Dépit amoureux Molière's. ${ }^{1}$ Es ist charakteristisch, dals es in B fehlt. An A I wurden A II und A III gefügt. Nach A III, B I der riote im engern Sinne gehen die Fortsetzungen auseinander, so dafs wir von den bis jetzt bekannten Stücken folgendes Schema entwerfen können:

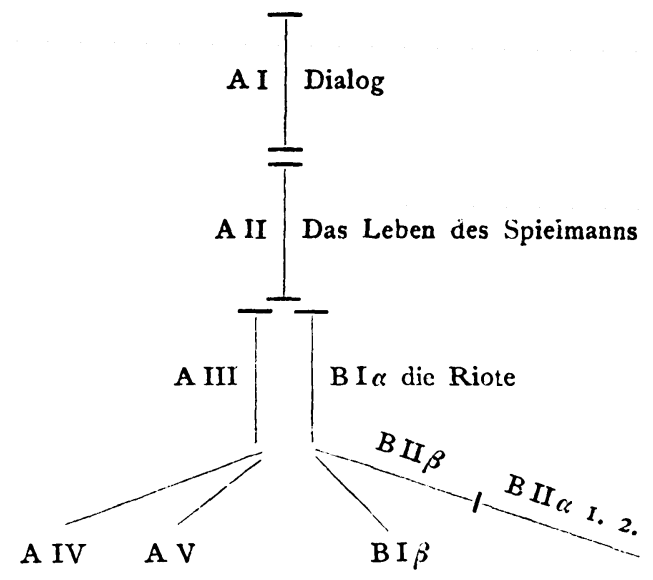

In Worten ausgedrückt: das Centrum ist die riote $d u$ monde. An diese werden in $\mathrm{A}$ oben zwei Stücke, unten je eins angefügt. In

1 L. Petit de Julleville, Les Mystères I 269. 
B findet blofs eine Verquickung mit andern, ursprünglich unabhängigen Stücken statt, und zwar auf der einen Seite mit einer weltlichen Ballade des vérités, auf der andern mit dem Preise eines gottesfürchtigen Lebens. In der ersten wird das Thema B I $\alpha$ noch etwas weiter geführt durch B I $\beta$. B II $\alpha$ war ursprünglich eine Ballade, deren Gegenstück in weit künstlerischer Form sich bei Villon unter dem Titel: La ballade des contrevérités findet. 1 Parallelen zu den Proverbes ethniques finden sich bei H. Gaidoz et P. Sébillot, Le Blason populaire de la France. Paris 1884 .

Der Leser wird bemerken, dals die sämtlichen Stücke für die Lexikographie sehr ausgiebig sind.

1 ed. Longnon S. 138.

J. ULRICH. 